\title{
EXISTENCE OF SOLUTIONS OF A NONLINEAR DIFFERENTIAL EQUATION
}

\author{
L. CESARI AND R. KANNAN ${ }^{1,2}$
}

\begin{abstract}
A criterion is proved for the existence of at least one solution to the equation $u^{\prime \prime}+u=g(u)+h$ with $u(0)=u(\pi)=0$, where $h \in L_{2}[0, \pi]$ and $g$ is continuous monotone nonincreasing.
\end{abstract}

In a number of papers [5-7] we have considered the problem of the periodic solutions (harmonics) of Liénard systems, and we have given sufficient conditions for the existence of such solutions in a number of rather general situations. This we have done by using variants of the alternative method (cf. also $[\mathbf{1}, \mathbf{2}, \mathbf{3}, \mathbf{4}, \mathbf{8}]$ ). We are interested here in the solutions of the nonlinear problem

$$
\begin{gathered}
u^{\prime \prime}+u=g(u)+h, \quad u(0)=u(\pi)=0, \quad|g(u)| \leqslant C_{1}+C_{2}|u|, \\
h \in L_{2}[0, \pi], \quad g \text { continuous and monotone, } \quad g(\infty)=\infty .
\end{gathered}
$$

A paper has recently appeared $[\mathbf{1 0}]$ in which the authors use a method which could be thought of as a variant of the alternative method we have used in [4-6] and show the existence of solutions of the nonlinear problem (1) under suitable growth hypotheses on $g(u)$ including oddness and a bound on $C_{2}$.

In the present paper we should separate the case of $g(u)$ nondecreasing from the case in which $g(u)$ is nonincreasing.

For $g$ nondecreasing neither oddness nor any bound on $g$ is needed. In an independent paper we discuss this case among other aspects of boundary value problems at resonance. Indeed, following our results in [12] and using the ideas in [5-9], we improve this case to essentially requiring that $g$ be eventually increasing and thereby improve on previous work in [11].

For $g$ nonincreasing, certainly a bound on $C_{2}$ is needed since for $C_{2}=3, C_{0}=0$, $g(u)=-3 u, h=\sin 2 t$, problem (1) has no solution. However, for this case, $g$ monotone nonincreasing by using the same method above, we prove Theorems (1.i) and (2.i) which seem to improve the results in [10] since oddness is not needed and the estimate $\gamma<0.443$ in (1.i) replaces a weaker estimate in [10] (cf. Remark 2 at the end of the present paper).

Received by the editors December 22, 1980 and, in revised form, November 30, 1981.

1980 Mathematics Subject Classification. Primary 34B15, 34C15, 34C25.

Key words and phrases. Resonance, alternative method, auxiliary and determining equations, LeraySchauder topological argument.

${ }^{1}$ The first author was partially supported by National Science Foundation Research Grant MCS 80-02337. The second author was partially supported by U. S. Army Research Grant DAAG 29-80-C-0060.

${ }^{2}$ The authors thank the referee for his remarks which contributed to the final wording of the paper. 
1. We first consider the following situation which seems to be relevant.

(1.i) Theorem. Let $h:[0, \pi] \rightarrow \mathbf{R}, h \in L_{2}[0, \pi], g: \mathbf{R} \rightarrow \mathbf{R}$ be continuous monotone nonincreasing with $|g(\xi)| \leqslant C+\gamma|\xi|$ for some constants $\gamma>0, C \geqslant 0$, and such that $\lim \sup _{\xi \rightarrow \infty}(-g(\xi) / \xi)=\gamma$ in the sense that for every $\varepsilon>0$ there are numbers $\bar{X}>0$ as large as we want such that $-g(\bar{X}) / \bar{X}>\gamma-\varepsilon$ and $-g(-\bar{X}) /(-\bar{X})>\gamma-\varepsilon$. Then, for $\gamma<0.443$ there is at least one solution $u(t), 0 \leqslant t \leqslant \pi$, to problem (1), $\left(u, u^{\prime}\right)$ absolutely continuous with $u^{\prime \prime} \in L_{2}[0, \pi]$.

Proof. Under the stated assumptions, we see that for every $\varepsilon>0$ there are also numbers $\bar{\xi}>0$ as large as we want such that $-g(\bar{\xi}) / \bar{\xi} \geqslant \gamma-\varepsilon,-g(-\bar{\xi}) /(-\bar{\xi}) \geqslant \gamma-\varepsilon$.

In terms of the alternative method (cf. e.g. [3]), we take $X=Y=L_{2}[0, \pi]$, $E u=u^{\prime \prime}+u$ for $u \in D[E]$ and boundary conditions $u(0)=u(\pi)=0$, so that the eigenvalues of $E$ are the integers $1-k^{2}$, and the corresponding normalized eigenfunctions are $(2 / \pi)^{1 / 2} \sin k t, k=1,2, \ldots$, and $\operatorname{ker} E=\{\sin t\}$.

Any element $u \in L_{2}[0, \pi]$ has Fourier series

$$
u=\sum_{1}^{\infty} a_{k}(2 / \pi)^{1 / 2} \sin k t, \quad a_{k}=\left(u,(2 / \pi)^{1 / 2} \sin k t\right),
$$

and we define the projection operators $P: X \rightarrow X, Q: Y \rightarrow Y$ by taking $P u=Q u=$ $a_{1}(2 / \pi)^{1 / 2} \sin t$. Then, for $X_{1}=(I-P) X, Y_{1}=(I-Q) Y$, and any element $u \in Y_{1}$, we define as usual $H: Y_{1} \rightarrow X_{1}$ by taking

$$
u_{1}=H u=-\sum_{2}^{\infty}\left(k^{2}-1\right)^{-1} a_{k}(2 / \pi)^{1 / 2} \sin k t
$$

for

$$
u=\sum_{2}^{\infty} a_{k}(2 / \pi)^{1 / 2} \sin k t \in Y_{1}
$$

Then

$$
\begin{gathered}
\|u\|=\left(\sum_{2}^{\infty} a_{k}^{2}\right)^{1 / 2} \\
\|H u\|=\left(\sum_{2}^{\infty}\left(k^{2}-1\right)^{-2} a_{k}^{2}\right)^{1 / 2} \leqslant 3^{-1}\left(\sum_{2}^{\infty} a_{k}^{2}\right)^{1 / 2}=3^{-1}\|u\| \\
\|H u\|_{\infty} \leqslant \operatorname{Sup}_{t}\left(\sum_{2}^{\infty}\left(k^{2}-1\right)^{-2}\right)^{1 / 2}(2 / \pi)^{1 / 2}\left(\sum_{2}^{\infty} a_{k}^{2} \sin ^{2} t\right)^{1 / 2} \\
\leqslant \mu\left(\sum_{2}^{\infty} a_{k}^{2}\right)^{1 / 2}=\mu\|u\|,
\end{gathered}
$$


where $\mu=(2 / \pi)^{1 / 2} \sigma$ and $\sigma=\left(\sum_{2}^{\infty}\left(k^{2}-1\right)^{-2}\right)^{1 / 2}$. It is easy to compute $\sigma$ by comparison with the well-known series $S_{4}=\Sigma_{1}^{\infty} k^{-4}=1.08232$. Indeed, for every $m$ we have

$$
\begin{aligned}
\sum_{2}^{m-1}\left(k^{2}-1\right)^{-2}+ & {\left[S_{4}-\sum_{1}^{m-1} k^{-4}\right]<\sigma^{2} } \\
& <\sum_{2}^{m-1}\left(k^{2}-1\right)^{-2}+\left(m^{4}\left(m^{2}-1\right)^{-2}\right)\left(S_{4}-\sum_{1}^{m-1} k^{-4}\right)
\end{aligned}
$$

and for $m=10$ we obtain $\sigma=0.36736$ and $\mu=0.29311$ (the error being $<10^{-5}$ ). With these notations, problem (1), or $E x=N x$, is equivalent to the system of auxiliary and determining equations

$$
u_{1}=K_{1} u \equiv H(I-P) N u, \quad K_{0} u \equiv Q N u=0,
$$

where $u=u^{*}+u_{1}, u^{*} \in X_{0}, u_{1} \in X_{1}$. We will now apply an abstract existence theorem derived in [13] to the above system of equations.

Here $N$ is continuous, $H(I-P)$ is compact; hence, $K_{1} u=H(I-P) N$ is compact. On the other hand, $K_{0}=Q N$ is continuous. Let us now prove that we can take $R_{0}, r>0$, so that $\left\|u^{*}\right\| \leqslant R_{0},\left\|u_{1}\right\| \leqslant r, u=u^{*}+u_{1}$ implies $\left\|K_{1} u\right\| \leqslant r$. For any $R_{0}, r>0$, let $\Omega$ denote the set $\Omega=S_{0} \times S_{1}$ with $S_{0}=\left[u^{*} \in X_{0} \mid\left\|u^{*}\right\| \leqslant R_{0}\right]$, $S_{1}=\left[u_{1} \in X_{1} \mid\left\|u_{1}\right\| \leqslant r\right]$.

First, we have

$$
\begin{aligned}
|(N u)(t)| & =|g(u)+h| \leqslant C+\gamma|u(t)|+|h(t)|, \\
\|N u\| & \leqslant C \sqrt{\pi}+\gamma\|u\|+\|h\|, \\
\left\|\bar{u}_{1}\right\| & =\|H(I-P) N u\| \leqslant 3^{-1} C \sqrt{\pi}+3^{-1} \gamma\left(R_{0}+r\right)+3^{-1}\|h\|,
\end{aligned}
$$

and thus $\left\|K_{1} u\right\| \leqslant r$ provided

$$
\begin{aligned}
\gamma<3, \text { and } & r \geqslant\left(1-3^{-1} \gamma\right)^{-1}\left(3^{-1} C \sqrt{\pi}+3^{-1} \gamma R_{0}+3^{-1}\|h\|\right), \text { or } \\
r & \geqslant p R_{0}+q, \text { with } \\
p & =\left(1-3^{-1} \gamma\right)^{-1}\left(3^{-1} \gamma\right), \quad q=\left(1-3^{-1} \gamma\right)^{-1}\left(3^{-1} C \sqrt{\pi}+3^{-1}\|h\|\right)
\end{aligned}
$$

We have just proved that $\left\|\bar{u}_{1}\right\| \leqslant r$ for $\left\|u^{*}\right\| \leqslant R_{0},\left\|u_{1}\right\| \leqslant r$. We shall now verify that

$$
\left(\operatorname{SQN}\left(u^{*}+\bar{u}_{1}\right), u^{*}\right) \leqslant 0[\geqslant 0]
$$

for all $u^{*} \in X_{0}, u_{1} \in X_{1},\left\|u^{*}\right\|=R_{0},\left\|u_{1}\right\| \leqslant r$. When this is done, we know by (2.i) of [13] that there is at least one point $u=u^{*}+u_{1} \in \Omega$ such that $u_{1}=K_{1} u$, $K_{0} u=0$, that is, $u=u^{*}+u_{1}$ is a solution of the original problem (1). Statement (2.i) is proved in [13] by the Leray-Schauder topological argument, and an alternative proof is also given in [13] in terms of Schauder's fixed point theorem. 
Let $g_{1}=-g$. Condition (4) in the present case becomes, for $\rho= \pm R_{0}$ and dropping the factor $\rho$,

$$
\begin{aligned}
I & =\int_{0}^{\pi}\left[g_{1}\left(\rho(2 / \pi)^{1 / 2} \sin t+\overline{u_{1}}(t)\right)-h(t)\right](2 / \pi)^{1 / 2} \sin t d t \\
& \geqslant 0 \text { for } \rho=R_{0},
\end{aligned}
$$

and $I \leqslant 0$ for $\rho=-R_{0}$. Let us see in detail the first case. For some $\eta, 0<\eta<$ $(2 / \pi)^{1 / 2}$, let $F_{0}=\left[t \in[0, \pi] \mid(2 / \pi)^{1 / 2} \sin t>\eta\right]$, and $F_{1}=[0, \pi]-F_{0}$. We have now

$$
\left|g_{1}\left(\rho(2 / \pi)^{1 / 2} \sin t+\overline{u_{1}}(t)\right)\right| \leqslant C+\gamma\left|\rho(2 / \pi)^{1 / 2} \sin t+\overline{u_{1}}(t)\right|, \quad t \in[0, \pi],
$$

where

$$
\|u\|=\left\|\rho(2 / \pi)^{1 / 2} \sin t+\overline{u_{1}}\right\| \leqslant\left(R_{0}^{2}+r^{2}\right)^{1 / 2},
$$

and hence

$$
\begin{aligned}
& \left\|g_{1}\left(\rho(2 / \pi)^{1 / 2} \sin t+\overline{u_{1}}(t)\right)\right\| \leqslant C \sqrt{\pi}+\gamma\left(R_{0}^{2}+r^{2}\right)^{1 / 2} \\
& \left\|\overline{u_{1}}\right\|_{\infty}=\left\|H(I-P)\left[g_{1}\left(\rho(2 / \pi)^{1 / 2} \sin t+\overline{u_{1}}(t)\right)-h\right]\right\|_{\infty} \\
& \quad \leqslant \mu\left(C \sqrt{\pi}+\gamma R_{0}+\gamma r+\|h\|\right) .
\end{aligned}
$$

Therefore, for $t \in F_{0}$ we also have

(6)

$$
\rho(2 / \pi)^{1 / 2} \sin t+\overline{u_{1}}(t) \geqslant \eta R_{0}-\mu \sqrt{\pi} C-\mu \gamma R_{0}-\mu \gamma r-\mu\|h\|=\chi, \quad t \in F_{0} .
$$

To make $\chi>0$, we require

$$
\begin{aligned}
\eta-\mu \gamma & >0, \quad \text { and } \quad(\eta-\mu \gamma) R_{0}>\mu \sqrt{\pi} C+\mu \gamma r+\mu\|h\|, \quad \text { or } \\
\eta-\mu \gamma & >0, \quad R_{0}>p_{1} r+q_{1}, \quad \text { with } \\
p_{1} & =(\eta-\mu \gamma)^{-1}(\mu \gamma), \quad q_{1}=(\eta-\mu \gamma)^{-1}(\mu \sqrt{\pi} C+\mu\|h\|) .
\end{aligned}
$$

We note that, for $0<\sigma<1, \sin t=\sigma, 0<t<\pi / 2$, we take $t=\arcsin \sigma=\sigma \kappa(\sigma)$, where $1<\kappa(\sigma)<\pi / 2=1.57079$ and $\kappa(\sigma) \rightarrow 1$ as $\sigma \rightarrow 0+$. Thus, for $0<\eta<$ $(2 / \pi)^{1 / 2}$ we have

$$
(2 / \pi)^{1 / 2} \sin t=\eta \quad \text { for } \sin t=(\pi / 2)^{1 / 2} \eta
$$

or

$$
t=(\pi / 2)^{1 / 2} \eta \kappa \quad \text { with } \kappa=\kappa\left((\pi / 2)^{1 / 2} \eta\right)=\left((\pi / 2)^{1 / 2} \eta\right)^{-1} \arcsin \left((\pi / 2)^{1 / 2} \eta\right)
$$

Also,

$$
\text { meas } F_{0}=\pi-2(\pi / 2)^{1 / 2} \eta \kappa, \quad \text { meas } F_{1}=2(\pi / 2)^{1 / 2} \eta \kappa
$$


with $\kappa=\kappa\left((\pi / 2)^{1 / 2} \eta\right)$. Finally, since $g_{1}$ is monotone increasing, we have

$$
\begin{gathered}
\int_{F_{0}}\left[g_{1}\left(\rho(2 / \pi)^{1 / 2} \sin t+\overline{u_{1}}(t)\right)\right](2 / \pi)^{1 / 2} \sin t d t \\
\geqslant\left(\pi-2(\pi / 2)^{1 / 2} \eta \kappa\right) \eta g_{1}(\chi) .
\end{gathered}
$$

On the other hand, for $t \in F_{1}$, we have

$$
\begin{aligned}
& \left|\rho(2 / \pi)^{1 / 2} \sin t+\overline{u_{1}}(t)\right| \leqslant R_{0} \eta+\mu\left(C \sqrt{\pi}+\gamma R_{0}+\gamma r+\|h\|\right), \quad t \in F_{1}, \\
& \left|g_{1}\left[\rho(2 / \pi)^{1 / 2} \sin t+\overline{u_{1}}(t)\right]\right| \\
& \leqslant C+\gamma R_{0} \eta+\mu \gamma C \sqrt{\pi}+\mu \gamma^{2} R_{0}+\mu \gamma^{2} r+\mu \gamma\|h\|, \quad t \in F_{1}, \\
& \text { (9) }\left|\int_{F_{1}}\left[g_{1}\left(\rho(2 / \pi)^{1 / 2} \sin t+\overline{u_{1}}(t)\right)\right](2 / \pi)^{1 / 2} \sin t d t\right| \\
& \quad \leqslant 2(\pi / 2)^{1 / 2} \kappa \eta^{2} \cdot\left(C+\gamma R_{0} \eta+\mu \gamma C \sqrt{\pi}+\mu \gamma^{2} R_{0}+\mu \gamma^{2} r+\mu \gamma\|h\|\right) .
\end{aligned}
$$

From the assumptions on $g_{1}$ we know that given $\varepsilon>0$, there are numbers $\bar{\chi}>0$ as large as we want such that $g_{1}(\bar{\chi}) \geqslant(\gamma-\varepsilon) \bar{\chi}$. Thus, if $\chi$, as defined by $(6)$, is one of these numbers, then by (6) and the monotonicity of $g_{1}$ we have, for $t \in F_{0}$.

$$
\begin{aligned}
g_{1}\left(\rho(2 / \pi)^{1 / 2} \sin t+\overline{u_{1}}(t)\right) & \geqslant g_{1}(\bar{\chi}) \\
& \geqslant(\gamma-\varepsilon)\left(\eta R_{0}-\mu \sqrt{\pi} C-\mu \gamma R_{0}-\mu \gamma r-\mu\|h\|\right) .
\end{aligned}
$$

Finally, from (5), (8), (9) we have

$$
\begin{aligned}
I= & \left(\int_{F_{0}}+\int_{F_{1}}\right) g_{1}\left(\rho(2 / \pi)^{1 / 2} \sin t+\overline{u_{1}}(t)\right)(2 / \pi)^{1 / 2} \sin t d t \\
& -\int_{0}^{\pi} h(t)(2 / \pi)^{1 / 2} \sin t d t \\
\geqslant & \left(\pi-2(\pi / 2)^{1 / 2} \eta \kappa\right) \eta(\gamma-\varepsilon)\left(\eta R_{0}-\mu \sqrt{\pi} C-\mu \gamma R_{0}-\mu \gamma r-\mu\|h\|\right) \\
& -2(\pi / 2)^{1 / 2} \kappa \eta^{2}\left(C+\gamma R_{0} \eta+\mu \gamma C \sqrt{\pi}+\mu \gamma^{2} R_{0}+\mu \gamma^{2} r+\mu \gamma\|h\|\right)-\|h\| \\
= & {\left[\left(\pi-2(\pi / 2)^{1 / 2} \eta \kappa\right) \eta(\gamma-\varepsilon)(\eta-\mu \gamma)-2(\pi / 2)^{1 / 2} \kappa \eta^{2}\left(\gamma \eta+\mu \gamma^{2}\right)\right] R_{0} } \\
& -\left(\pi-2(\pi / 2)^{1 / 2} \eta \kappa\right) \eta(\gamma-\varepsilon)(\mu \sqrt{\pi} C+\mu \gamma r+\mu\|h\|) \\
& -2(\pi / 2)^{1 / 2} \kappa \eta^{2}\left(C+\mu \gamma \sqrt{\pi} C+\mu \gamma^{2} r+\mu \gamma\|h\|\right)-\|h\| .
\end{aligned}
$$

To make $I>0$, first we require the coefficient $m$ of $R_{0}$ to be positive, hence

$$
m=\left(\pi-2(\pi / 2)^{1 / 2} \eta \kappa\right) \eta(\gamma-\varepsilon)(\eta-\mu \gamma)-2(\pi / 2)^{1 / 2} \kappa \eta^{2}\left(\gamma \eta+\mu \gamma^{2}\right)>0,
$$

and by taking $\alpha=2(\pi / 2)^{1 / 2} \kappa, \varepsilon=0$, and dividing by $\eta \gamma$, we require

$$
(\pi-\alpha \eta)(\eta-\mu \gamma)>\alpha \eta(\eta+\mu \gamma), \text { or } \eta-\mu \gamma>(2 \alpha / \pi) \eta^{2} \text {. }
$$


In other words, not only do we need $\eta-\mu \gamma>0$ as required in (7), but we also need $\eta-\mu \gamma>(2 \alpha / \pi) \eta^{2}$ or, equivalently, $\eta-(2 \alpha / \pi) \eta^{2}>\mu \gamma$. The first member takes its maximum value for $\eta=\eta_{0}=\pi / 4 \alpha$, and thus $\gamma$ must be below the corresponding value, or $\gamma<\gamma_{0}=\mu^{-1} \eta_{0}\left(1-(2 \alpha / \pi) \eta_{0}\right)=\pi / 8 \alpha \mu=(2 \mu)^{-1} \eta_{0}$. By taking note of the value of $\alpha$ we also have

$$
\eta_{0}=\pi / 4 \alpha=\pi\left(8(\pi / 2)^{1 / 2} \kappa\right)^{-1}=\left(\pi \eta_{0} / 8\right)\left(\arcsin \left((\pi / 2)^{1 / 2} \eta_{0}\right)\right)^{-1},
$$

and we see that this relation determines $\eta_{0}$, since $0<\eta_{0}<(2 / \pi)^{1 / 2}$ and $\arcsin \left((\pi / 2)^{1 / 2} \eta_{0}\right)=\pi / 8$. For $\arcsin c=\pi / 8=0.39269$, we then have $c=$ $\sin (0.39269)=0.38267$, so that $\kappa=(0.39269)(0.38267)^{-1}=1.02618$, and then

$$
\eta_{0}=(\sqrt{2} / \sqrt{\pi})(0.38267)=0.30533, \quad \alpha=\left(2(\pi / 2)^{1 / 2} \kappa\right)=2.57224,
$$

and

$$
\gamma_{0}=(0.30533) \cdot 2^{-1}(0.29311)^{-1}=0.52084, \quad 0<\gamma<\gamma_{0} .
$$

We see that we can take $\eta=\eta_{0}$ and then $0<\gamma<\gamma_{0}$, so that (12) holds. Hence, we can take $\varepsilon>0$ sufficiently small so that (11) holds, that is, the coefficient $m$ of $R_{0}$ in the estimate (10) of $I$ is positive. For $\varepsilon=\varepsilon^{\prime} \gamma$, we also have from (11):

$$
\begin{aligned}
m & =(\pi-\alpha \eta) \eta \gamma(\eta-\mu \gamma)-\alpha \eta^{2}\left(\gamma \eta+\mu \gamma^{2}\right)-\varepsilon^{\prime}(\pi-\alpha \eta) \eta \gamma(\eta-\mu \gamma) \\
& =\eta \gamma\left[\pi(\eta-\mu \gamma)-2 \alpha \eta^{2}-\varepsilon^{\prime}(\pi-\alpha \eta)(\eta-\mu \gamma)\right]>0 .
\end{aligned}
$$

Then, for the positivity of $I$, we require from (10) that

$$
\begin{aligned}
& \eta \gamma\left[\pi(\eta-\mu \gamma)-2 \alpha \eta^{2}-\varepsilon^{\prime}(\pi-\alpha \eta)(\eta-\mu \gamma)\right] R_{0} \\
& \quad \geqslant \eta \gamma\left[(\pi-\alpha \eta)\left(1-\varepsilon^{\prime}\right) \mu \gamma+\alpha \eta \mu \gamma\right] r \\
& \quad+\eta \gamma\left[(\pi-\alpha \eta)\left(1-\varepsilon^{\prime}\right)(\mu \sqrt{\pi} C+\mu\|h\|)+\alpha \eta\left(\gamma^{-1} C+\mu \sqrt{\pi} C+\mu\|h\|\right)\right]+\|h\|,
\end{aligned}
$$

or

$$
R_{0} \geqslant p_{2} r+q_{2},
$$

with

$$
\begin{aligned}
p_{2}= & {\left[\pi(\eta-\mu \gamma)-2 \alpha \eta^{2}-\varepsilon^{\prime}(\pi-\alpha \eta)(\eta-\mu \gamma)\right]^{-1}(\mu \gamma)\left[(\pi-\alpha \eta)\left(1-\varepsilon^{\prime}\right)+\alpha \eta\right], } \\
q_{2}= & {\left[\pi(\eta-\mu \gamma)-2 \alpha \eta^{2}-\varepsilon^{\prime}(\pi-\alpha \eta)(\eta-\mu \gamma)\right]^{-1} } \\
& \times\left[(\pi-\alpha \eta)\left(1-\varepsilon^{\prime}\right)(\mu \sqrt{\pi} C+\mu\|h\|)\right. \\
& \left.+\alpha \eta\left(\gamma^{-1} C+\mu \sqrt{\pi} C+\mu\|h\|\right)+(\eta \gamma)^{-1}\|h\|\right] .
\end{aligned}
$$

Relations (3), (7) and (14) require that

$$
\begin{aligned}
& R_{0}>p_{1} r+q_{1}>p_{1} p R_{0}+\left(p_{1} q+q_{1}\right), \\
& R_{0}>p_{2} r+q_{2}>p_{2} p R_{0}+\left(p_{2} q+q_{2}\right) .
\end{aligned}
$$


Hence, we require $p_{1} p<1, p_{2} p<1$, and

$$
R_{0}>\left(1-p_{1} p\right)^{-1}\left(p_{1} q+q_{1}\right), \quad R_{0}>\left(1-p_{2} p\right)^{-1}\left(p_{2} q+q_{2}\right),
$$

and we require $r$ to satisfy (15), which now implies (3).

We now have

$$
\begin{aligned}
p_{1} p= & (\eta-\mu \gamma)^{-1}(\mu \gamma)\left(3^{-1} \gamma\right)\left(1-3^{-1} \gamma\right)^{-1}, \\
p_{2} p= & {\left[\pi(\eta-\mu \gamma)-2 \alpha \eta^{2}-\varepsilon^{\prime}(\pi-\alpha \eta)(\eta-\mu \gamma)\right]^{-1} } \\
& \times \mu \gamma\left[(\pi-\alpha \eta)\left(1-\varepsilon^{\prime}\right)+\alpha \eta\right]\left(3^{-1} \gamma\right)\left(1-3^{-1} \gamma\right)^{-1} .
\end{aligned}
$$

To guarantee that $p_{1} p<1, p_{2} p<1$, we first take $\varepsilon^{\prime}=0$ so that these requirements reduce to

$$
\mu \gamma\left(3^{-1} \gamma\right)<(\eta-\mu \gamma)\left(1-3^{-1} \gamma\right), \text { or } \quad\left(3^{-1} \eta+\mu\right) \gamma-\eta<0,
$$

and

$$
\mu \gamma(\pi)\left(3^{-1} \gamma\right)<\left(\pi(\eta-\mu \gamma)-2 \alpha \eta^{2}\right)\left(1-3^{-1} \gamma\right)
$$

or

$$
\left(3^{-1} \eta+\mu\right) \gamma-\eta<0, \quad\left[3^{-1} \eta(\pi-2 \alpha \eta)+\pi \mu\right] \gamma-\eta(\pi-2 \alpha \eta)<0 .
$$

We have $\mu=0.29311$, and we take $\eta=\eta_{0}=0.30533$, so that

$$
\begin{gathered}
\kappa=1.02612, \quad \alpha=2.57224, \quad 3^{-1} \eta+\mu=0.39488, \quad \pi-2 \alpha \eta=1.57083, \\
3^{-1} \eta(\pi-2 \alpha \eta)+\pi \mu=1.08070, \quad \eta(\pi-2 \alpha \eta)=0.47962, \\
\left(3^{-1} \mu\right)(2 \alpha \eta)=0.15347,
\end{gathered}
$$

and the inequalities above become, respectively,

$$
(0.39488) \gamma-0.30533<0, \quad(1.08070) \gamma-0.47962<0 .
$$

Hence, $\gamma<\gamma_{11}=0.77322$, and $\gamma<\gamma_{21}=0.4438$, the latter restriction being stronger than the former, and also stronger than the two others, $\gamma<3$ and $\gamma<\gamma_{0}=0.52084$, we met before. We take $\gamma=0.443$. By choosing $\varepsilon$, that is, $\varepsilon^{\prime}>0$ sufficiently small, we have $p_{1} p<1, p_{2} p<1$, and (3), (7) and (14) reduce to (15) and (16).

It is enough that we now take $R_{0}>0$ sufficiently large and in such a way that the corresponding $\chi$ as given by (6) is one of the numbers $\bar{\chi}$ which are now determined since we have already chosen $\varepsilon=\gamma \varepsilon^{\prime}>0$. For $\rho=-R_{0}$ the same considerations hold with (6) replaced by

$\rho(2 / \pi)^{1 / 2} \sin t+\overline{u_{1}}(t) \leqslant-\eta R_{0}+\mu \sqrt{\pi} C+\mu \gamma R_{0}+\mu \gamma r+\mu\|h\|=-\chi, \quad t \in F_{0}$.

By the statement (2.i) of [13] we conclude that there is some $u=u^{*}+u_{1} \in \Omega$ such that $u_{1}=K_{1} u, K_{0} u=0$. This completes the proof of (1.i). 
2. We shall consider here another situation which also seems to be of interest.

(2.i) Theorem. Let $h:[0, \pi] \rightarrow \mathbf{R}, h \in L_{2}[0, \pi]$ and let $g: \mathbf{R} \rightarrow \mathbf{R}$ be continuous monotone with $|g(\xi)| \leqslant C+D|\xi|$ for all $\xi \in \mathbf{R}$ and some constants $C \geqslant 0$ and $0<\gamma \leqslant D$, and such that $\lim \sup _{\xi \rightarrow \infty}(-g(\xi) / \xi)=\gamma$ in the same sense as in Theorem (1.i). Then, for any $0<\gamma<0.443$, there is a constant $\Gamma=\Gamma(\gamma)>0.443$, such that, for $\gamma \leqslant D<\Gamma(\gamma)$, problem (1) has at least a solution $u(t), 0 \leqslant t \leqslant \pi$.

For $D=\gamma$ this theorem reduces to Theorem (1.i).

Proof. Let $D=\theta \gamma$ for some $\theta \geqslant 1$. Let us proceed as in $\S 1$, where now in the estimates we shall need $D=\theta \gamma$ instead of $\gamma$, while the estimates where we have $\gamma-\varepsilon$ remain the same. The final result is that the problem has solutions if we can satisfy certain inequalities, where now $\theta$ appears together with $\gamma$. For $\theta=1$ these inequalities reduce to those of $\S 1$, and we have seen that the latter could be satisfied in the strong sense by taking $\eta, \kappa, \alpha$ as in $\S 1$ and $\gamma<0.443$. By continuity argument there is some $\theta_{0}>1$, depending on $\gamma$, such that the same inequalities are satisfied for all $\theta, 1 \leqslant \theta \leqslant \theta_{0}$.

REMARK 1. (1.i) and (2.i) do not hold for $\gamma=0$. Indeed, for $g \equiv 0, h=\varepsilon \sin t$, $\varepsilon>0$, problem (1) has no solution (no matter how small $\varepsilon$ is).

However, if $g$ is continuous in $\mathbf{R}$ with $\|g(\xi)\| \leqslant M$ for $\xi \in \mathbf{R}$ (and thus $\gamma=0$ ), and if there are constants $c<d, C<D$ such that $g(\xi) \leqslant C$ for $\xi \leqslant c, g(\xi) \geqslant D$ for $\xi \geqslant d$, and $\left|\int_{0}^{\pi} h(t) \sin t d t\right|<D-C$, then there is a solution $x(t), 0 \leqslant t \leqslant \pi$, to problem (1).

This is the essence of the Lazer and Leach theorem [9], which is restated here in the present situation. The proof, on the same idea as the present paper, is given in $[3$, p. 144] and can be repeated in the present case.

REMARK 2. Statement (1.i) seems to improve those in [10] where it is required that $g$ be odd (we do not need this assumption) and $\gamma<0.24347$ (we require $\gamma<0.443$ ). For instance, for $g(\xi)=1+3^{-1} \xi$, for $\xi>0, g(\xi)=e^{\xi}+3^{-1} \xi$ for $\xi \leqslant 0$, and $h(t)=$ $\sin t$, problem (1) has a nonzero solution.

\section{REFERENCES}

1. L. Cesari, Functional analysis and periodic solutions of nonlinear differential equations, Contributions to Differential Equations, Wiley, New York, 1963, pp. 149-187.

2. Functional analysis and a reduction method, Michigan Math. J. 11 (1964), 385-414.

3. Functional analysis, nonlinear differential equations, and the alternative method, Nonlinear Functional Analysis and Differential Equations (L. Cesari, R. Kannan, J. D. Schuur, eds.), Dekker, New York, 1976, pp. 1-197.

4. __ A nonlinear problem in potential theory, Michigan Math. J. 16 (1969), 3-20.

5. L. Cesari and R. Kannan, Periodic solutions in the large of nonlinear differential equations, Rend. Mat. Univ. Roma (2) 8 (1975), 633-654. (1976), 101-124. , Solutions in the large of Lienard systems with forcing terms, Ann. Mat. Pura Appl. (4) 111

7. (6) 2 (1982), 217-223.

8. J. K. Hale, Applications of alternative problems, Lecture Notes, Brown Univ., Providence, R.I., 1971.

9. A. C. Lazer and D. E. Leach, Bounded perturbations of forced harmonic oscillations at resonance, Ann. Mat. Pura Appl. 72 (1969), 49-68. 
10. M. Schechter, J. Shapiro and M. Snow, Solutions of the nonlinear problem $A u=N u$ in a Banach space, Trans. Amer. Math. Soc. 241 (1978), 69-78.

11. P. J. McKenna and J. Rauch, Strongly nonlinear perturbations of nonnegative boundary value prohlems with kernel, J. Differential Equations 28 (1978), 251-265.

12. L. Cesari and R. Kannan, Functional analysis and nonlinear differential equations, Bull. Amer. Math. Soc. 79 (1973), 1216-1219.

13. __ Solutions of nonlinear hyperbolic equations at resonance, Nonlinear Anal. 6 (1982), 751-805.

Department of Mathematics, University of Michigan, Ann Arbor, Michigan 48104

Department of Mathematics, University of Texas at Arlington, Arlington, Texas 76019 